\title{
Neuroblastoma en la infancia
}

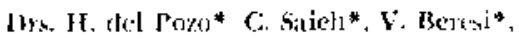 \\ J. f. 1 atomat** y J. Rajuric***
}

F. neuroblastoma es un tumor embrionario derivado de la cresta neural, que aparece en mís die la mitad de los casos en las cípsulas suplarrenales o en los ganglios simpáticos de la pirte alta abdominal. Los restannes tiencn crigen en cualquier parte do la cotcha simpítica. Las metástasis se hacen por vía linfática y sanguinca; los ganglios linfáticos, el sistema esqueletico y cl higado son los mís frecuentemente afectados y sólo en un $10 \%$ se hallan en los pulmones.

Histológicamente se caracterina por cóluhs de 10 a 15 micrones de dímetro, compuestas casi enteramente por núcleos hipercromáticos, citoplasmat escaso, de variadas formas, adoptando algunas veces el aspecto de ncurofibri1las. pero que se diferencian por no teñirse con métolos atgénticos. Se observa la formación de rosetas sólo en un $20 \%$ dé los casos. Existen variaciones cehulares en los diferentes tumores, adoptando algunos, caractercs más inmaturos que otros, pero sin embargo la evolución $y$ el pronistico son exactamente los mismos $(29-23)$. Es importante sefialar que el diañóstico histológico debe ser complementado con histoquŕmica y si es posible con cultivo tumoral. La tendencia actual es no hacer diferenciaciones entre los grados histológicos del neuroblastoma (25).

A pesar que es uno de los tumores de ma-

\footnotetext{
- Centro alc Tumores. Hospital Calvo Mackenna.

- 1)epartatuento de Anatomia Patológica. Hospital Calvo Mackenna.

**Radioterapeuta. Fundación A. López Pérez.
}

Rev. Chilena de Pediatrfa, vol. 47, No J, 1976 yor malignidad en la infancia su proniostico es improdecible y es conocida su regresión espontánea en ausenciat de terapia (16).

Lin nuestro país el neuroblastom: es un tumor relativamente poco frecuente. En la estarlistion del Centro de Tumores del I lospital Luis Calvo Mackenna, ocupa el tereer Jugar cntre los tunoress sólidos maliynos, a $60 \mathrm{~m}$ tinuacion de low linfomas $y$ nefrohlastomits. En Fs ados linidos de N. A., Ios fumeres mi:ligno: jumto con la leucemia, ocupan sl segundo lugar dentro de las causas de muerte, luego de los accidente; y en algunos paises es el tumor sólido maligno más frecuente en la infancia (1-2-5) .

El objetivo de éstit presentitción es mostrar lin experienciát sobre 12 casos reunidos en un perículo de 6 años.

\section{MATERIAL I' METODOS}

Se analizan 12 niños con el díngnóstico de neuroblastoma, atendidos en el Cuntro de Oncología, entre enero 1968 y dicicmbre 1973.

En el momento del ingreso del enfermo se realiza una historia y examen físico completo y se solicitan los siguientes exámenes de latboratorio: Henograma, Mielograma, Orina, V.H.S. P.P.D. 2U, Haptoglobinas, Gatecolaminas y estudio radiológico que comprende tórax, abdomen, esqueléto y pielografía. Con estos elementos se sospecha el diagnóstico; si el timor se considera extirpable se procede a la resección completa, si es muy grande se 
hate quinioterapia previa con el objeto de reducir su tamaño para extirparlo posteriormente en fornia completa. En caso de no encontrarse el tumor primitivo se procede a efectuar biopsia de las nretástasis.

Se confirma el diagnóstico con el estudio histepatologico del tumor y se realiza la clitsificución clinica (Tabla No 1) cuyo objetivo es decidir el tipo de tratamiento y formular um pronóstico $(7)$. Fxisten varias clásificaciones que presentan variantes más o menos importantes, pero en esta casuística se tsa lia presen. tarla por Pinckel (14).

Tabla 1

CLASIFICACION DE I.OS NEUROBLASTOMAS (Pinckel)

T. Lacal completametite resecable

II. Regional

a) resecable pircjalsetente

b) no resceable.

III. Sistenico

a) sin comproniso medular

b) con invasión micitular

El tratamiento de estos enfermos se hace con radioterapia y quimioterapia. Es muy importante para el aclecuado tratamiento radian. te, como en todo tumor infantil o de adulto, una cuidadosa planificación que inclaya torla el área afectada, no sobrepasándola innecesíriamente.

En la presente serie se utilizó la Cobaltoterapia sobre la zona de ubicación die la lesión original o de las metástasis comprobadas histopatológicamente. Las dosis suministradas fueron de 3.150 a 4.500 rads., dauas en un lapso de 4 a 5 semanas, excepto en un caso en que el tratamiento se prolongó par 81 días debido a marcada Ieucopenia. En uno de los pacientes en que se comprobó la presencia de infiltración hepática se irradió la zona operatoria y el hígado con una dosis de 3.150 rads. En otro se hizo irradiación intensiva, paliativa en días con 2.000 rads., por las pésimas condiciones generales.
La quiomioterapia se ha efectuado en base a dos esquemis dilerentes: secuencial, usa(i) basta 1970 y posteriormente, simulánes, que se usi laasta la fecha.

Ouinioterapia secuencial. Se uso radiotesipiit primero, luego ciclofosfamicla I.V. duJinte 5 días en dosis de $10 \mathrm{mg} / \mathrm{K} / \mathrm{c} / \mathrm{dia}$ continuando en forma oral clurante 30 días $(5 \mathrm{mg}$ ! $\mathrm{Ko} / \mathrm{di} t)$. Despues vincrisina, con una dosis semanal hasta completar 4 dosis comenzando con $0.050 \mathrm{mg} / \mathrm{Kg}$, atumentando on $0.025 \mathrm{mg} /$ $\mathrm{Kg}$ closis hasta llegar a $0,125 \mathrm{mg} / \mathrm{Kg}$.

Estas curas se alternaron con períodos de descanow de 3 meses. Lat duración total de lit guimioterapia fue de 2 años.

Quimioterapia simultinea, o actual. Se hace radiotcrapia concomitantemente con quimiotertpia. Ciclofosfamida $200 \mathrm{mg}$, por $\mathrm{m}^{2}$ de superficie corporal y vincristina $\mathbf{l}, 4 \mathrm{~m}_{\text {; }}$ por $\mathrm{m}^{2}$ cle supurlicie corporal por dosis.

En el estado 1 se inclica vincristina y ciclofoslamicla semanal durante 6 meses. En los restantes extados se usan ambats drogas en forma semanil durante 6 meses y luego cada J'1 clías Jasta completar 2 años.

\section{RESULTADOS}

La distribución por edad y sexo se muestra en la figura 1. El período prediagnóstico, vale decir, el ticmpo transcurriclo entre 13 iniciación de los síntomas y el diagnóstico, varió entre 9 clías $y$ I año 8 meses.

La ubicación del tumor correspondió en $\tilde{s}$ casos a la región abdominal: 3 se situaron en

DISTRIBUCION SEGUN EDAD Y SEXO

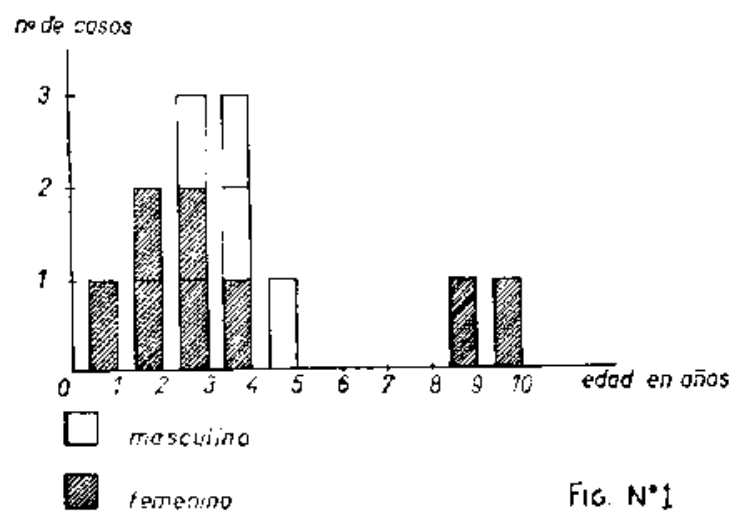


lis capwulas suprarrenales y 2 en ganglios sim1'áticos retroperitoneales.

En 3 niños la presentación fue toríxica. en 1 fue cervical y en $y$ no se encontró el tumor original (Tabla 2).

Tabla Ṅ\% 2

SOBREVIDA Y LOCALVZACION INICIAL. DE 12 CASOS DE NECROBLASTOMA

(Centro de Oncologia, Hoxpitul I., Calvo Mackenna) (1968-1978)

\begin{tabular}{lccc}
\hline Localización & $\lambda^{\prime} \dot{ }$ casus & Faliecidos & Fivos \\
\hline & 5 & 1 & 4 \\
Abdominal & 3 & 1 & 2 \\
Coríxica & 1 & - & 1 \\
Cervical & 3 & 2 & 1 \\
Desconocida & & \\
\hline
\end{tabular}

En la Tabla 3 se analizan los signos y síntomas iniciales más frecuentes.

Los F.P.D. fueron positivos en 2 de los pacientes, Ia V.I.S. y los valores de haptoglobina fueron variables en cada uno de los ninos.

Tablia $\mathrm{XO}_{3}$

\section{SINTOMAS Y SIGNOS INICIALES}

DE II CASOS DE NELIROBIASTOMA

(Centro de Oncologia, Hosp. I. Calvo Mackenniz) (1968-1975)

\begin{tabular}{|c|c|}
\hline Tumor & 8 \\
\hline Disminuución die peso & 8 \\
\hline Metitstasis óseas & 5 \\
\hline Fiebric & g \\
\hline Vomitos & 2 \\
\hline Hotor alxtominal & 2 \\
\hline Paraplejia & 2 \\
\hline Disurteda & 1 \\
\hline Artralgias & $\mathbf{l}$ \\
\hline Palidez & $\mathbf{1}$ \\
\hline Adtunopatias & $\mathbf{l}$ \\
\hline Rectorragia & l \\
\hline
\end{tabular}

El miclograma efectuado en 10 cle los 12 niños reveló invasión medular en 4 y en los restantes se mantuvo reiteraclamente normal.

La radiografía de Tórax no mostró com. promiso mediastínico, excepto en los 3 casos que se iniciaron como tales.

En 4 pacientes la pielografía señaló alteraciones conpatibles con el diagnóstico.

La radiografia de esqueleto mostró alteraciones en 5 niños, 3 de estos en cráneo, en uno simultáneamente en cráneo, tibia y costillas $y$ otro en humero $y$ femur.

En ajgunos casos se realizó determinación de catecolaminas, prueba que se realizó en disLintos laboratorios y con técnicas diferentes lo que no permitió sacar conclusiones y súlo nos limitamos a mencionar este hecho.

La relación entre frecuencia y escado se puede observar en la Tabla 4. En la misma se puede ver que hay 8 pacientes vivos y 4 fallecidos. La sobrevida en los primeros fluctúa entre 2 y 55 meses (mediana 13,5 meses) $y$ en los fallecidos es entre 5 y 9 meses (me. diana 5 meses).

Tabla $\mathrm{YO}_{4}$

\section{REIAACION ENTRE ESTADO, EDAD, SOBREVIDA \\ Y TRATAMIENTO EN I2 CASOS DE NEUROBLASTOMA}

(Centro de Oncologia, Hosp. L. Calvo Mackenna) (1968-1973)

\begin{tabular}{|c|c|c|c|}
\hline Estado & Edaul & Sobrevida & Tratamiento \\
\hline $\mathbf{I}$ & 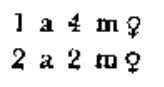 & $\begin{array}{l}\operatorname{l~} \mathrm{m}^{*} \\
\operatorname{la~} 4 \mathrm{m*}\end{array}$ & $A$ \\
\hline IIa & - & - & - \\
\hline $11 b$ & 9 a $10 \mathrm{~ms}$ & $2 \mathrm{~m}$ & $A$ \\
\hline $111 a$ & $\begin{array}{l}3 \text { an } 9 \\
1 \text { a } 9 \mathrm{mq} \\
5 \text { a } 2 \mathrm{~m} \\
8 \text { a } 8 \\
9 \text { a } 5 \mathrm{mo}\end{array}$ & $\begin{array}{l}4 \text { a } 3 \mathrm{~m} \\
6 \mathrm{~m} \\
4 \text { a } 7 \mathrm{~m} \\
9 \mathrm{~m}^{* *} \\
1 \text { a } 5 \mathrm{~m}^{*}\end{array}$ & $\begin{array}{l}\mathbf{S} \\
\mathbf{A} \\
\mathbf{S} \\
\mathbf{S} \\
\mathbf{A}\end{array}$ \\
\hline IIII & $\begin{array}{l}2 \text { a } 4 \mathrm{~m}+ \\
2 \text { a } 5 \mathrm{~m} \text { क } \\
3 \text { a } 9 \\
3 \text { a } 10 \mathrm{~ms}\end{array}$ & $\begin{array}{l}5 \mathrm{~m} * * \\
4 \mathrm{~m} \\
5 \mathrm{~m} * \\
5 \mathrm{~m} *\end{array}$ & $\begin{array}{l}A \\
A \\
S \\
S\end{array}$ \\
\hline
\end{tabular}

- Sin eviklencia de enfermedad.

**Fallecidos.

S Tratamiento secuedcial.

A Tratamiento actual o simultáneo. 


\section{COMENTARIO}

Sexo: En Ia serie estudiada, el $58 \%$ de los pacientes fueron mujeres y el $41,6 \%$ hombres y no hubo diferencias estadísticamente significativa en cuanto a mortalidad, lo que concuerda con otras publicaciones $(8-9)$.

Edad: El rango, de edad varió entre los 3 meses y los 9 años 5 meses. La mayoría fueron menores de 4 años (figura $\mathrm{N} \%$ I) un $50 \%$ de los casos, se ubicó entre los 2 y 3 años de cdad, cifras señaladas por algunos autores (8-10). Evahs sostiene que el $82 \%$ de los niños menores de 1 año sobreviven micntras que to hace súlo el $10 \%$ de los niños con 2 años o más. Ẽr muestra casuística los 6 menores de 2 años están vivos, en cambio pasados los 2 años sobreviven 2 niños de 6 .

El periodo pretiagnóstico no tiene relación con la extensión del proceso ni con el pronós. tico y creemos que el diagnóstico precoz no es real, ya que pueden cxistir perfodos asintomáticos prolongados jor ser tumores profundos que pasan desapereibidos, lo que se confirma en 3 de nuestros casos en los que el diagnós. tico se hizo sólo por la aparición de las me. tástasis. Según Evahs (15) la mortalidad es mayor en estos casos y asi lo confirmamos en nuestra serie, ya que de Ios 4 pacientes fallecidos, en 2 no se encontró el tumor original (Tabla 2).

Como factor pronóstico se da además la "bicación del tumor. La presentación cervical o torácica tendrfa mayor sobrevida (15), pero en los casos nuestros en 3 de 4 niños que fallecieron, hubo compromisos torácico.

De acuerdo con V.G. Stella (24) el compromiso esquelético está en intima relación con el pronóstico y es así como en todos los casos fallecidos de la serie hubo metátasis óseas. En cambio las metátasis hepáticas son de mejor pronóstico.

Por Jas radiografías de tórax se descartó en todos los casos metátasis pulnoonares, lo que está de aucerdo con los estudios clásicos.

Con la pielografía se demostró en $\mathbf{5}$ casos rechazo renal, semejando el movimiento de abertura de un abanico (26); no se encontró calcificaciones así como tampoco distorsión de
Ios calices como se observa en los nefroblastomas y que permite hacer diagnóstico diferencial (2-11).

De los 4 casos con invasión medular, es decir estado mb, 3 fallecieron y el actualmente sobreviviente tiene 2 meses de evolución con tratamiento, pero es portador de metástasis óseas, es mayor de 2 años y la respuesta clínica no es buena, de lo que resulta un mal pronós. tico.

En relación a V.H.S. y Haptoglobinas, hay estrecha correlación entre amhos valores, en la medida que uno desciende lo lace el otro. En todo caso una determinación aislada de haptoglobinas no nos sirve como índice pronóstico ya que para ello necesitanos una curva, $y$ si sus valores son mantenidamente altos, nos habla de mal pronóstico.

Koop y col (16) refieren la regresión espon. tánea en individuos portadores de neuroblastoma lo que se lia comprobado en ninguno de los 12 casos estudiados. Así también Be. ckith y Mortin (17) dicen que el grado de diferenciación del tumor influye en el pronós. lico, pero en Ios casos de la serie la histopato. logía no la mostrado grados marcados de diferenciación. Tampoco se lia observado una relación clínica histologica tan estrecha como la vista en el retinoblastoma donde Ia existencia de Rosetas se asocia con un mejor pronóstico evolutivo.

Según D. Pinckel y col (14) la combinación de vincristina y ciclofosfamida prolonga la sobrevida en comparación con los pacientes que recibieron en forma exclusiva agentes alquilantes. Nosotros no podemos sacar conclusiones respecto a los tratamientos efectuados ya que el número de casos es pequeño y el periodo de observación es muy corto.

En base a estas consideraciones cabe pensar que aún no se puede hablar de indices pronósticos absolutos hasta no conocer perfectamente la histopatología de esta neoplasia y su constitución bioquimica, siendo esta última la más importante, puesto que controla el comportamiento de la célula neoplásica. Tumores que parecen iguales histopatológicamente no lo son en sus carácterfsticas quimicas lo que 
lakce que tengan patologia variable y respuesta imprevisible al tratamiento (3).

\section{RESUMEN}

Se revisan doce niños con diagnóstico de Neuroblastoma, estucliados en el Centro de Oncologia del Hospital "Luis Calvo Mackenni" entre los años 1968 y 1973 . Se usaron dos esquemas de tratamiento diferentes que incluian quimioterapia y radioterapia.

Se hizo cirugia en todos los casos, si el tumor era muy grande se realizó quimioterapia previa para reducir su tamaño, y de los casos en que no se encontró tumor se bizo biopsia de las metástásis. No lubbo diferencia en cuan. to a sexo, $y$ en cuanto a etlatd la mayoría de los casos se ubican entre los dos y cuatro años. En 5 casos la Jocilización fue abdominal, en tres torácica, en un niño la ubicación fue cervical y en tres no se encontró tumor primitivo.

De los 12 pacientes fallecen 4, todos mayores de 2 años y hay 2 con sobrevida superior a cuatro años y sin evidencias de enfermedades.

\section{SUMMARY}

Twelve children with Neuroblastoma treated at the Oncology Department at the Calvo Mackenna Children's Hopital during 1968. 1973 are reported.

Mixed treatment was used according to two difference schemes including chermotherapy and $X$. Ray.

$\mathrm{All}$ of them underwent surgery and with previous chermotherapy in order ti reduce the tumor size in those patients with very large tumors.

In these cases were no tumor was found, methastasis biopsy was performed.

There was no difference between sex and the mayority were 2 to 4 years old.

Abdominal location of the primitive tumor was found in 5 cases, thoracic in 3, cervical in 1 of them and uncentain in 3 patient.

The ever-all mortality was $39 \%$ (4/12) all of them above 2 years old, 2 patient have a survival more than 4 years and without evidence of disease at present. 